\title{
Les pompes primaires des réacleurs PWR
}

\section{Francis Ruyan}

Société JEUMONT-SCHNEIDER

Dans les réacteurs nucléaires à eau pressurisée, une circulation d'eau est établie entre le cœur du réacteur formé par le combustible et les générateurs de vapeur. Ce circuit constitue ce qui est couramment appelé le "circuit primaire". Cette circulation d'eau est assurée par les pompes primaires. Elle réalise le refroidissement des assemblages de combustible dans la cuve du réacteur et transfère ensuite les calories à des échangeurs de vapeur pour produire dans le circuit secondaire la vapeur qui alimente la turbine.

Les réacteurs PWR comportent généralement 3 ou 4 boucles reliées à la cuve et composées chacune d'un générateur de vapeur et d'une pompe primaire.

- La figure 1 montre l'arrangement d'une boucle

- La figure 2 schématise un réacteur à 3 boucles.

Leur rôle de moteur du fluide primaire caloporteur confère aux pompes primaires une importance essentielle pour le fonctionnement et la sécurité de la Centrale. C'est le souci d'assurer le meilleur service qui a présidé à la conception de ces pompes.

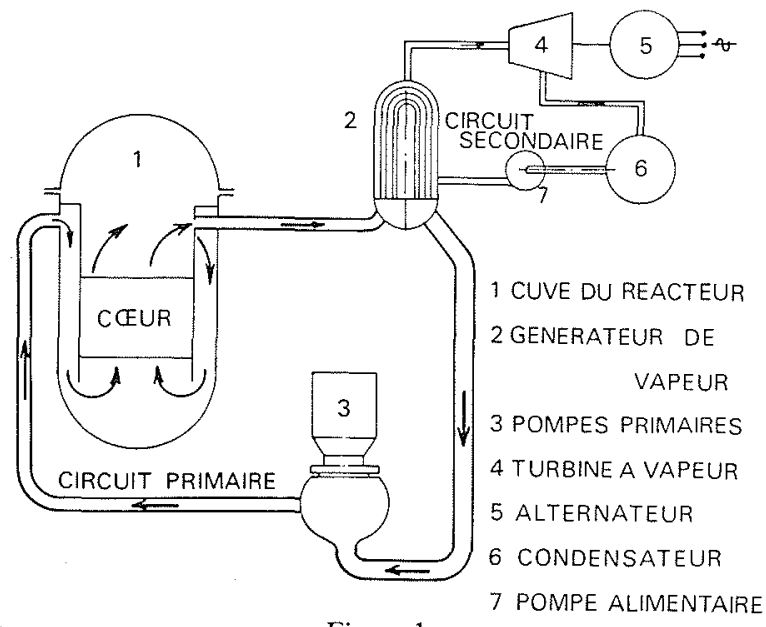

Figure 1

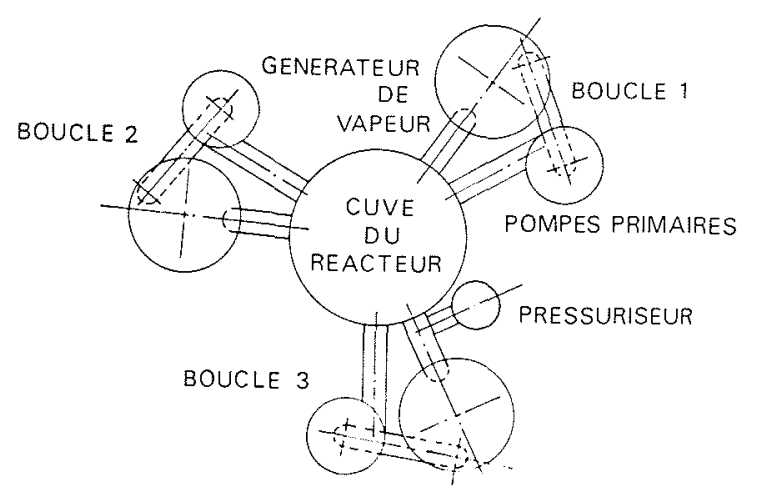

Figure 2

\section{Principales caractéristiques des pompes primaires actuelles}

Avant d'aller plus avant, afin de nous fixer les ordres de grandeur, examinons, à titre d'exemple, les principales caractéristiques des pompes primaires qui équipent les 24 réacteurs PWR du programme nucléaire français 900 MW actuellement en cours de réalisation ou de préparation.

Ces réacteurs, produisant sur le réseau une puissance électrique nette de $900 \mathrm{MWe}$, comportent trois boucles et par conséquent trois pompes primaires. Chacune d'elles assure un débit de $22000 \mathrm{~m}^{3} / \mathrm{h}$ sous une hauteur manométrique de $90 \mathrm{~m}$ d'eau. Leur vitesse de rotation est de $1485 \mathrm{tr} / \mathrm{mn}$.

Elles sont entrainées par un moteur électrique asyn. chrone à démarrage direct de $5600 \mathrm{KW}$. L'inertie totale du groupe motopompe est de $3800 \mathrm{~m}^{2} \mathrm{Kg}$. 


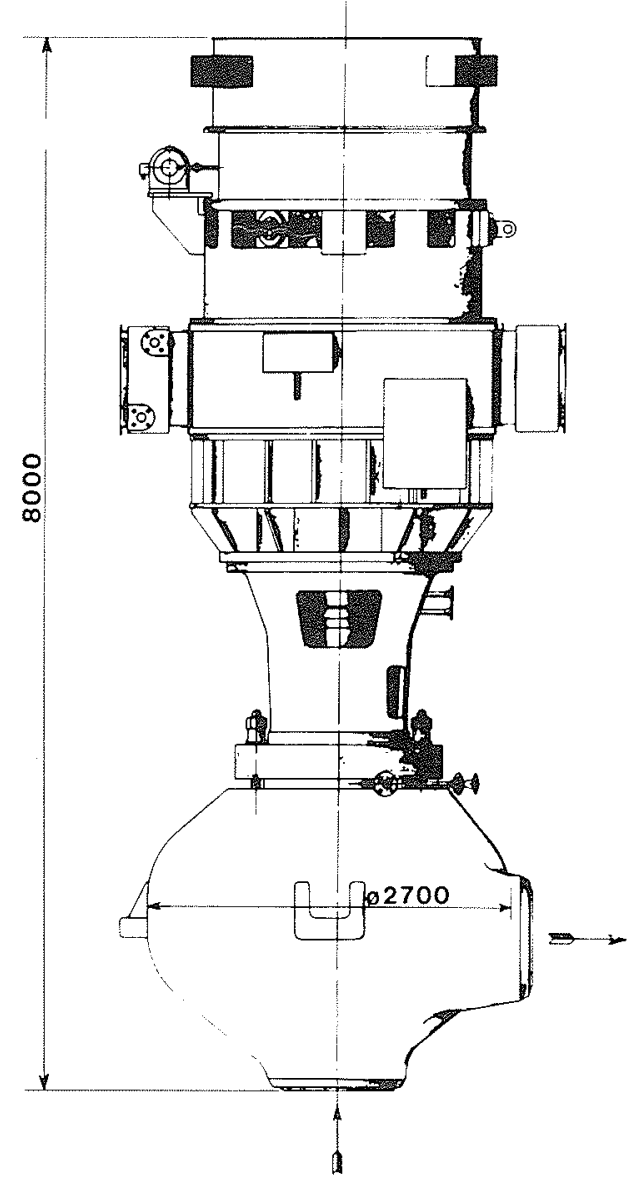

Figure 3

Le fluide véhiculé par les pompes primaires des réacteurs PWR se trouve à une température de l'ordre de 280 à $300^{\circ} \mathrm{C}$ sous une pression de 150 à 160 bars à l'aspiration de la pompe. L'eau du circuit primaire est additionnée d'acide borique qui permet d'ajuster la réactivité du cœur en fonction de l'usure du combustible. La teneur en acide borique varie entre quelques centaines et $4000 \mathrm{ppm}$ de bore en fonctionnement normal.

Ces groupes motopompes à axe vertical ont une hauteur totale d'environ $8 \mathrm{~m}$ et un diamètre de $2,7 \mathrm{~m}$ au niveau du corps de pompe, la masse totale de cet ensemble étant d'environ 85 tonnes (Fig. 3).

\section{Conception des pompes primaires}

Les pompes primaires de réacteur PWR ont un caractère très spécifique. Outre leur fonction hydraulique d'assurer la circulation du fluide primaire, ces pompes sont soumises à de nombreux impératifs de différents ordres qui conditionnent très fortement leur conception comme par exemple :

aspect mécanique : les pompes font partie de l'enceinte du circuit primaire soumise à forte pression et température; aspect environnement : elles doivent être capables de supporter, sans ruine de nature à compromettre la sécurité, les effets des séismes ou de l'accident de référence, ce qui influe sur l'architecture des groupes; L'eau véhiculée est radioactive ce qui requiert la limitation des débits de fuite à la sortie de l'arbre hors du corps de pompe;

aspect fiabilité de fonctionnement : l'accès limité dans le bâtiment du réacteur où se trouvent installées les pompes augmente le degré d'importance du bon fonctionnement mécanique;

aspect maintenance : celle-ci doit se faire sur des pièces radioactives qui doivent pouvoir être éventuellement décontaminées; et le temps d'intervention doit être le plus réduit possible.

Il existe une très grande interdépendance entre le tracé hydraulique et la conception des autres éléments $\mathrm{du}$ groupe motopompe. Par suite, la solution optimale du point de vue hydraulique pur ne peut pas être recherchée ou appliquée de la même façon que pour les pompes industrielles.

La conception des pompes primaires du réacteur PWR constitue en fait le résultat d'un compromis entre différentes contraintes qui sont d'ordre hydraulique, mécanique et de sécurité.

Du point de vue hydraulique, ces pompes primaires sont caractérisées par des vitesses spécifiques élevées de l'ordre de 120 à 140 . La vitesse de rotation de la pompe est choisie la plus élevée possible,compte-tenu des considérations mécaniques, de façon à réduire l'encombrement du groupe motopompe avec ses répercussions sur le génie civil du bâtiment du réacteur, et par suite permettre également une réduction du coût d'investissement de la centrale.

On constate que la valeur des vitesses spécifiques a tendance à croître au fil des projets, car, avec l'accroissement des tailles des réacteurs, les débits augmentent progressivement, alors que les hauteurs manométriques restent sensiblement du même ordre de grandeur ou du moins varient beaucoup moins vite. Ainsi par exemple les pompes primaires de la centrale nucléaire de Chooz, premier réacteur PWR industriel construit en France dans les années 62 à 65 , avaient un débit de $6000 \mathrm{~m}^{3} / \mathrm{h}$ sous une hauteur de $53 \mathrm{~m}$; les pompes des centrales actuellement en construction ont un débit environ 3,7 fois plus élevé tandis que leur hauteur manométrique n'a varié que dans un rapport de 1,7 . Il en résulte un accroissement de vitesse spécifique de $30 \%$.

La roue de pompe est une roue à un seul étage du type hélicocentrifuge adaptée pour assurer les caractéristiques hydrauliques demandées.

Autre point à noter sur l'aspect hydraulique : le problème de N.P.S.H. et de cavitation ne se pose pas avec ce type de pompe, car en fonctionnement normal la pression à l'aspiration de la pompe est de 155 bars alors que la tension de vapeur à $300^{\circ} \mathrm{C}$ n'est que de 80 bars environ.

La forme du corps de pompe est dictée par différentes considérations sortant du domaine hydraulique.

Il est de plus en plus souhaité d'avoir une tubulure de refoulement radiale, ce qui permet un meilleur agen- 
cement de l'intérieur du bâtiment du réacteur où la place est comptée. Mais vu les débits importants des pompes, cette solution n'est pas favorable du point de vue hydraulique.

Toutes les pièces en contact avec le fluide primaire sont réalisées en acier inoxydable et doivent avoir des surfaces lisses avec un très bon état de surface de façon, d'une part à éviter l'entraînement des particules métalliques par l'eau dans le cœur du réacteur, d'autre part à réduire la fixation de contamination radioactive sur les parois et faciliter la décontamination si nécessaire. Ceci conduit donc à avoir une forme intérieure de corps de pompe usinable au tour, c'est-à-dire un profil intérieur axisymétrique. Si toutefois les corps des premières pompes primaires PWR de la centrale de Chooz avaient une forme en colimaçon à section progressive, l'appropriation de surface étant faite par un meulage poussé, ceci n'est plus envisageable sur les pompes actuelles vu leur dimension et la surface mise en œuvre.

La forme du corps de pompe est également déterminée par le calcul des contraintes de pression et contraintes thermiques engendrées dans la pièce, ainsi que par l'application des règles de sureté qui deviennent de plus en plus précises et sévères.

Le corps de pompe formant un récipient soumis à forte pression, on cherche à se rapprocher de la forme sphérique qui est celle du solide de meilleure résistance mécanique. Dans le tracé du profil du corps de pompe on s'astreint aussi à réduire les contraintes thermiques induites par les différents transitoires pendant le fonctionnement du réacteur.

Il faut noter que le corps des pompes primaires pour les réacteurs PWR 900 MWe représente une masse de 32 tonnes d'acier inoxydable. Vu l'importance de ce poids, le corps est coulé en 2 ou 3 pièces assemblées par des soudures électroslag.

Les règlements de sureté requièrent maintenant une inspection en service des soudures de construction des enceintes nucléaires sous pression. C'est-à-dire que tous les 10 ans, il faut inspecter les soudures dans leur masse.

Du fait de la matière du corps de pompe et des épaisseurs mises en cuvre, ce contrôle ne peut se faire dans l'état actuel des techniques que par examen au bétatron ou accélérateur linéaire, examen qui est très peu pratique si non irréalisable à l'intérieur du bâtiment du réacteur.

Aussi pour les nouveaux modèles de pompes primaires qui équiperont les réacteurs PWR du palier $1300 \mathrm{MWe}$, nous sommes nous efforcés d'éliminer les soudures de construction, en étudiant les formes du corps de pompe pour qu'il puisse être réalisé en une seule pièce de fonderie.

L'inspection en service impose également un examen périodique de la surface intérieure du corps de pompe. Après fonctionnement de la centrale, cette surface devient assez radioactive ; il faut donc prévoir un accès facile au moyen d'endoscope ou périscope pour faire cet examen.

Cette condition, alliée à l'objectif, vu précédernment, de supprimer au maximum les soudures de construction sur les enceintes sous pression, nous a amené à remplacer la disposition initiale du diffuseur soudé à l'intérieur sur le corps de pompe, par une solution de diffuseur amovible pour les derniers modèles de pompes primaires.

Mais cet arrangement impose que le diamètre maximal du diffuseur soit limité à l'alésage de la bride supérieure du corps de pompe qui ne peut pas raisonnablement être augmenté exagérément sans avoir des répercussions sur les taux de contraintes et les renforcements des systèmes de boulonnage. De façon à avoir une longueur de diffusion suffisante, nous sommes donc pratiquement conduits à un tracé de diffuseur à écoulement axial.

\section{Evolution de la conception des pompes primaires}

Les pompes primaires véhiculant de l'eau radioactive, le problème de la réduction et du contrôle des fuites est important.

Les premiers réacteurs PWR industriels qui furent construits entre les années 1955 et 1965 et qui correspondaient à des puissances de centrale de 100 à $300 \mathrm{MWe}$, furent équipés de pompes primaires à moteur chemisé. La conception de ces pompes, formant un groupe monobloc à axe vertical avec le moteur d'entraînement, assure une étanchéité parfaite grâce à l'élimination de tout joint tournant ou presse-étoupe. En effet, les étanchéités sont ramenées à des étanchéités par joints statiques entre les différentes pièces du groupe motopompe.

La figure 4 représente une coupe de principe de pompe à moteur chemisé. La cavité intérieure du moteur

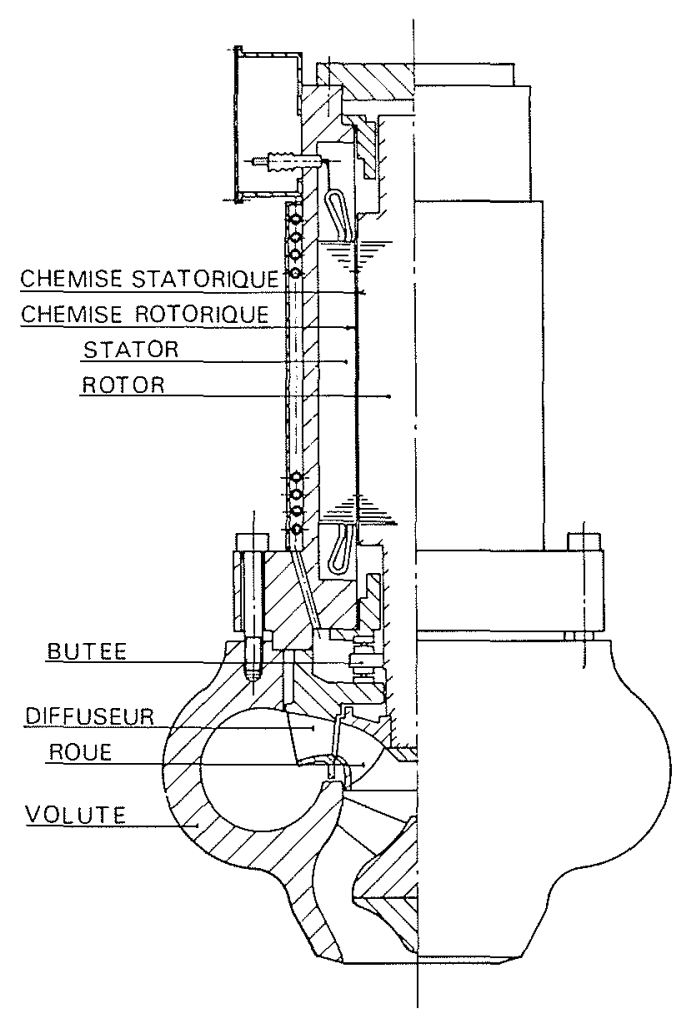

Figure 4 
est en communication avec la volute et se trouve donc remplie d'eau du circuit primaire maintenue froide par une barrière thermique et un réfrigérant.

Les composants électriques de la machine, son rotor et son stator, sont toutefois séparés de l'eau sous pression par une chemise, cylindre métallique de faible épaisseur en métal amagnétique qui s'appuie sur les tôles du circuit magnétique.

La centrale de Sena à Chooz, premier réacteur PWR industriel construit en France, est ainsi équipée de 4 pompes primaires de ce type assurant chacune un débit de $6000 \mathrm{~m}^{3} / \mathrm{h}$.

Vu sa grande sécurité, et sa simplicité d'exploitation due à la réduction des auxiliaires, ce type de pompe est celui qui équipe encore pratiquement tous les réacteurs de propulsion navale.

Parallèlement à l'accroissement des tailles des réacteurs nucléaires qui ont maintenant des puissances de 900 à $1200 \mathrm{MWe}$, les pompes primaires atteignent des débits unitaires dépassant $20000 \mathrm{~m}^{3} / \mathrm{h}$ et l'augmentation de leurs puissances fait que la solution des pompes à moteur chemisé n'est plus adaptée.

En cas de coupure générale de l'alimentation électrique, les pompes primaires doivent continuer, pendant les premières minutes qui suivent, à assurer un débit suffisant à travers le cœur du réacteur pour empêcher la fusion du combustible et enlever la chaleur résiduelle dégagée par les produits de fission. Vu la taille des réacteurs, ces règles de sécurité conđuisent à concevoir les groupes motopompes avec une inertie propre extrêmement élevée, inertie qui est obtenue par l'adjonction d'un volant sur la ligne d'arbre. Cette solution nécessite la sortie de l'arbre à travers l'enceinte sous pression.

Par ailleurs, le développement de la technologie des joints d'arbre pour circuits sous forte pression a permis d'atteindre maintenant un degré de fiabilité acceptable et une périodicité de révision compatible avec celles du changement de combustible.

Ces différents facteurs ont conduit à une nouvelle conception des pompes primaires pour réacteur PWR de puissance : les pompes primaires à joints d'arbre avec fuite contrôlée et limitée.

\section{Description des pompes primaires à joint d'arbre à fuite contrôlée}

La figure 5 présente l'architecture générale des pompes primaires à joint d'arbre qui équipent les réacteurs PWR français du niveau $900 \mathrm{MWe}$.

Ces pompes se composent essentiellement des 4 parties suivantes:

- La partie hydraulique

- Le système d'étanchéité le long de l'arbre

- Le moteur d'entraînement

- La butée et le volant.

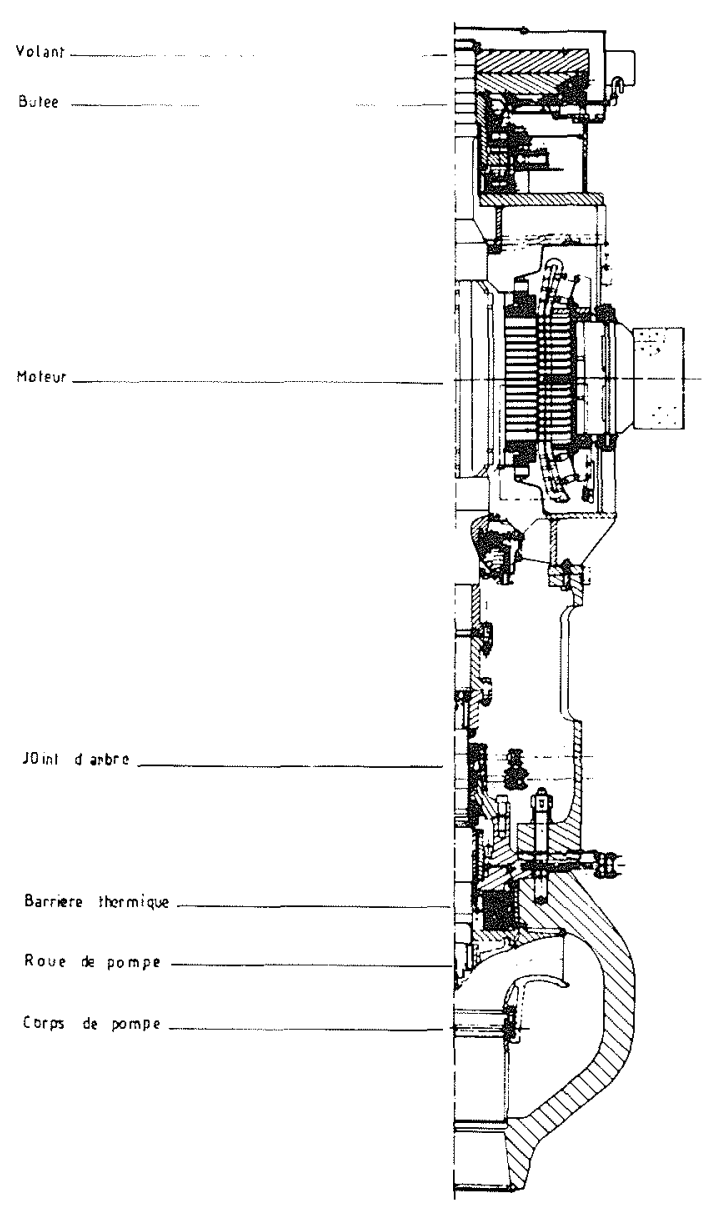

Figure 5 - Pompe primaire type 93D 7

\section{Partie hydraulique}

A la partie inférieure du groupe, nous trouvons le corps de pompe qui est soudé directement aux tuyauteries du circuit primaire par l'intermédiaire de ses tubulures.

Dans le cas des pompes primaires des réacteurs $900 \mathrm{MW}$, le diffuseur est soudé à l'intérieur du corps de pompe sur sa bride supérieure. Un guide d'eau s'emmanchant dans le flasque inférieur du diffuseur prolonge, dans le corps de pompe, le canal d'aspiration jusqu'à l'ouie de la roue.

La roue hélicocentrifuge à un étage est emmanchée sur une portée conique à l'extrémité de l'arbre pompe.

\section{Barrière thermique et dispositif d'étancheité}

Au-dessus de la roue se trouve la barrière thermique qui empêche le flux de chaleur dû à l'eau chaude de la pompe de remonter vers les parties supérieures, c'està-dire vers le palier et le système de joints d'arbre.

Cette barrière thermique est constituée d'un échangeur à serpentins dans lequel circule l'eau froide d'un circuit auxiliaire général de refroidissement.

Dans la bride de barrière thermique est installé le palier de la pompe qui est un palier à rotule lubrifié à l'eau. 
Nous trouvons ensuite le système d'étanchéité le long de l'arbre, qui assure la barrière de pression entre le circuit primaire et la pression atmosphérique.

Etant donné la pression élevée du circuit primaire, les joints de forme conventionnelle à surfaces frottantes sont mal adaptés. C'est donc un joint de type hydrostatique à fuite contrôlée qui est utilisé. Les faces du joint ont un profil spécial conçu pour garder un jeu constant et par suite une fuite contrôlée et limitée entre les deux faces.

Ce joint est suivi de deux joints à surfaces frottantes fonctionnant dans les conditions normales sous faible $\Delta P$.

Toutefois, le joint intermédiaire est prévu pour pouvoir supporter, pendant un certain temps, la pleine pression du circuit primaire en cas de défaillance du premier joint.

Les demi-accouplements de l'arbre pompe et l'arbre moteur sont reliés par l'intermédiaire d'un tronçon d'arbre démontable de façon à permettre les inspections périodiques des joints d'arbre sans devoir enlever le moteur.

\section{Moteur d'entrainement}

La pompe est entraînée par un moteur électrique asynchrone à cage à démarrage direct, alimenté en 6600 Volts.

Bien que ce moteur soit d'un type se rapprochant des moteurs classiques, il nécessite une conception particulière du fait de l'inertie importante demandée au groupe motopompe. Le moteur est réalisé avec un diamètre de rotor le plus grand possible compatible avec la tenue des matériaux.

Le refroidissement du moteur est assuré par circulation d'air ambiant, mais des réfrigérants à eau sont installés sur le circuit de retour d'air de façon à ne pas évacuer les pertes du moteur dans l'air de la salle du réacteur. Cette solution permet de limiter la ventilation du bâtiment, tout en profitant du volant thermique que représente le volume d'air du bâtiment en cas de coupure de l'eau de refroidissement.

\section{Butée et volant}

Au-dessus du moteur se trouve la butée double du type Kingsburry, à patins auto-équilibrés, prévue pour reprendre des efforts de l'ordre de 50000 daN.

En marche normale, la haute pression du circuit primaire appliquée sur l'arbre produit une poussée vers le haut qui est de loin supérieure à la poussée hydraulique de la roue de pompe et au poids du rotor.

Le volant d'inertie est installé à la partie supérieure de l'arbre de façon à permettre un accès facile pour les contrôles par ultrasons et examens par ressuage au cours des inspections périodiques en cours d'exploitation.

Le volant est équipé à sa partie inférieure d'un dispositif d'antidévirage. Celui-ci est constitué de cliquets venant s'insérer dans une roue à rochets lorsque la pompe non alimentée a tendance à tourner en sens inverse sous l'effet des autres pompes en marche.

La figure 6 donne une coupe schématique des pompes primaires qui équiperont prochainement les réacteurs nucléaires PWR à 4 boucles du palier $1300 \mathrm{MWe}$.

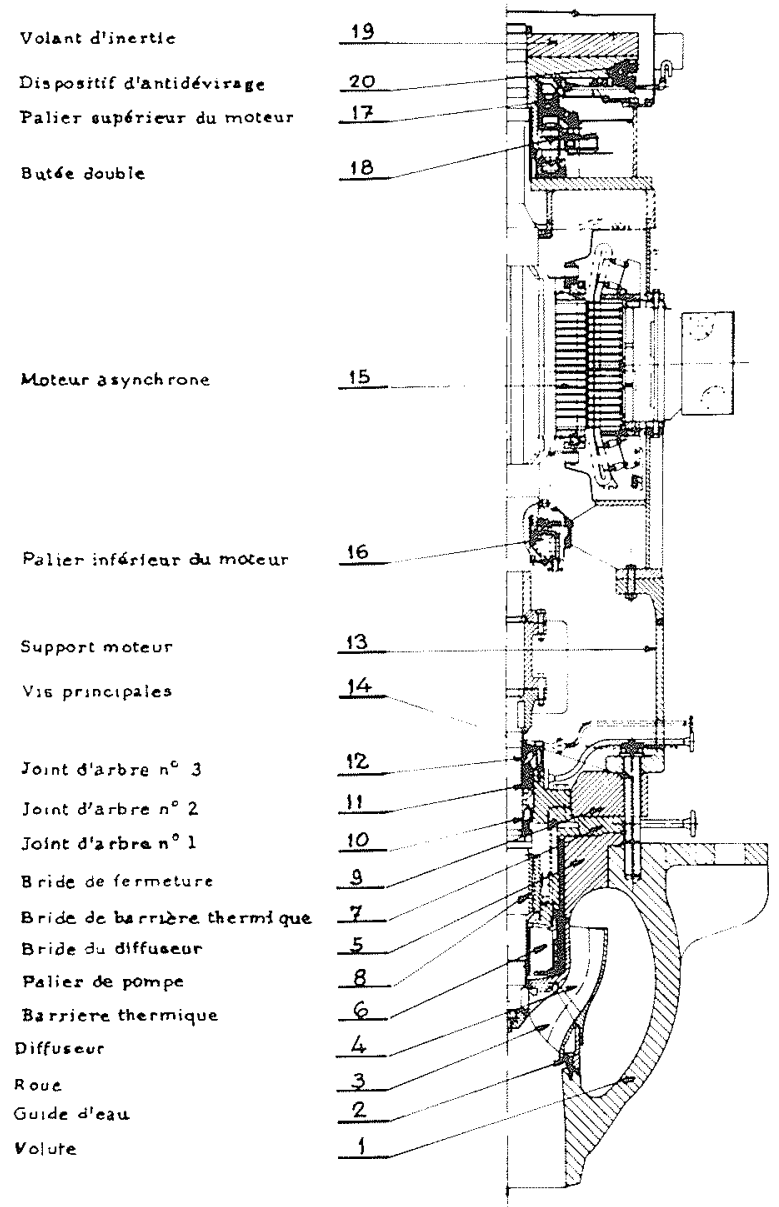

Figure 6 - Pompe primaire type 100

Ces pompes ont des caractéristiques hydrauliques plus poussées : elles doivent assurer un débit unitaire de 23000 à $24000 \mathrm{~m}^{3} / \mathrm{h}$ avec une hauteur manométrique de $100 \mathrm{~m}$.

Tout en conservant l'architecture générale des pompes précédentes, la conception de ces pompes pour les réacteurs $1300 \mathrm{MW}$ a été modifiée en vue de réduire au maximum les soudures sur l'enceinte sous pression, d'améliorer l'accessibilité de celles qui subsistent pour l'inspection en service, et de satisfaire les nouvelles exigences des règlements de sécurité.

Avant leur expédition sur site, les pompes sont soumises en usine à un programme d'essai sur boucle d'essai à débit réduit où sont reproduites les conditions de fonctionnement en centrale : température, pression..., excepté le débit.

Ces essais à débit réduit ont pour but de vérifier le comportement mécanique du groupe et le bon fonction. nement du système d'étanchéité sous différentes pressions et températures. Ils simulent également un certain nombre de situations exceptionnelles pouvant se produire au cours de l'exploitation de la centrale.

Ce programme d'essai à débit réduit est complété, pour la première pompe de chaque type, par des essais effectués en collaboration avec l'E.D.F. sur la boucle à plein débit installée à la Centrale de Gennevilliers. 
En conclusion, comme l'a montré ce rapport, les groupes motopompes primaires de réacteur PWR forment un ensemble dont les différents éléments : hydrauliques, mécaniques, électriques, sont absolument interdépendants et ne peuvent être étudiés séparément.
Dans la conception des pompes primaires, les problèmes de fiabilité et bon fonctionnement sont primordiaux et doivent passer avant la recherche de l'optimum des performances hydrauliques ou électriques.

\section{Discussion}

Président : M. P. BERGERON

M. le Président remercie M. PETIGNY de son exposé particulièrement clair et bien illustré. Il ouvre la discussion par la remarque suivante:

Ainsi que M. RUYANT l'a signalé dans son mémoire, l'évolution de la forme des pompes pour réacteurs a été largement influencée, sinon imposée, par des contraintes d'encombrement, de résistance mécanique et même d'économie, qui ont relégué parfois au second plan, la préoccupation de réaliser un écoulement hydraulique optimum. Alors qu'à l'origine les pompes des réacteurs P.W.R. comportaient un diffuseur, une volute, etc., elles deviennent, aujourd'hui, purement axiale et leur corps tend à devenir sphérique (pour obtenir une bonne résistance mécanique), tandis que leur zone d'aspiration et de refoulement est confinée dans un espace de plus en plus réduit et encombré. Ces contraintes, qu'il faut bien accepter, conduisent à une certaine diminution des performances hydrauliques des pompes, et en particulier, de leur rendement.

M. GILMER s'interroge sur les raisons qui ont fait assembler par boulonnage le diffuseur sur le corps de la pompe, décrite par le conférencier, alors que l'assemblage par soudure a été généralement utilisé même pour les brides d'aspiration et de refoulement. Peut-être a-t-on voulu ainsi faciliter le calcul de la résistance du système aux efforts engendrés par le séisme. C'est là, en effet, un problème difficile en raison de la grande Iongueur des arbres et la répartition des masses le long de ceux-ci.
Il ne s'agit pas d'un problème de tenue aux séismes, précise M. PETIGNY. Le boulonnage visé par M. GILMER a été adopté pour rendre le diffuseur démontable afin de permettre de contrôler et d'inspecter facilement - à l'endoscope - les soudures du corps de pompe, lequel fait partie de l'enceinte sous pression.

M. BRAZZINI s'inquiète de la diminution progressive des rendements tolérée pour ce genre de pompe; il en résulte une augmentation de l'énergie dissipée à l'intérieur de la pompe qui, au-delà d'une certaine limite, pourrait poser quelques problèmes.

Le rendement maximum pour les pompes actuelles de 900 MW est tout de même de 0,83 , ce qui limite le risque signalé, estime M. PETIGNY.

D'après les plans et coupes que vous avez montrés, remarque M. CANAVELIS, il semble que les entre-fers entre la roue et les diffuseurs sont assez faibles. De ce fait, ne retrouve-t-on pas dans ce type de pompe le problème des fluctuations de pression importantes, que l'on a évoqué hier, pour les turbines ou pour les pompes alimentaires; ces fluctuations engendrent des poussées radiales fluctuantes.

Des essais ont été faits à Chatou sur la pompe de Tihange, qui est très analogue au groupe que je vous ai présenté. On n'a rien signalé d'anormal, mais je ne peux répondre de façon précise à la question posée par M. CANAVELIS. 